\title{
Immunogenicity and Efficacy of a Rough Brucella suis Vaccine Delivered Orally or Parenterally to Feral Swine
}

\author{
S. C. Olsen ${ }^{1 *}$, P. Boggiatto ${ }^{1}$, J. Wilson-Welder ${ }^{1}$, P. Nol' ${ }^{2}$, J. Rhyan ${ }^{2}$ and N. Srirangathan ${ }^{3}$ \\ ${ }^{1}$ Infectious Bacterial Diseases of Livestock Research Unit, National Animal Disease Center, Agricultural Research Service, United States Department of \\ Agriculture, Ames, IA \\ ${ }^{2}$ Wildlife Livestock Disease Investigations Team, National Wildlife Research Center, Animal and Plant Health Inspection Service, Veterinary Services, United \\ States Department of Agriculture, Fort Collins, Colorado, USA \\ ${ }^{3}$ Department of Biomedical Sciences and Pathobiology, Virginia Maryland, College of Veterinary Medicine, Blacksburg, Virginia, USA
}

Received: 11 January, 2017; Accepted: 31 January, 2017; Published: 09 February, 2017

*Corresponding authors: Steven C. Olsen, NADC, USDA, ARS, 1920 Dayton Ave., Ames, IA 50010; Tel no: 515-337-7230; Fax No: 515

337-7458; E-mail: steven.olsen@usda.ars.gov

\begin{abstract}
Brucella suis strain $353-1$ is a stable vaccine strain that is clinically safe, does not cause positive serologic responses on conventional brucellosis surveillance tests, and induces humoral and cellular immunity in swine after vaccination. In this study, we evaluated tissue clearance and immunologic responses after oral or parenteral vaccination of feral swine with $1.9 \times 10^{10}$ colony-forming units (CFU) of strain 353-1, and compared efficacy of vaccination and control treatments in protecting against infection after experimental conjunctival challenge with a virulent $B$. suis strain. Feral swine vaccinated orally or parentally with strain 353-1 had greater $(P$ $<0.05$ ) mean ELISA titers to Brucella at all sampling times after vaccination when compared to non-vaccinated swine. PBMC from swine parentally vaccinated with $353-1$ at 12 or 17 weeks, or oral vaccinates at 12 weeks after vaccination, demonstrated greater $(\mathrm{P}$ $<0.05$ ) antigen-specific proliferative responses when compared to responses of PBMC from non-vaccinates. At necropsy, 4 weeks after experimental challenge with virulent $B$. suis, non-vaccinated feral swine had greater standard tube agglutination titers and disseminated infection with higher $(P<0.05)$ colonization $(\mathrm{CFU} / \mathrm{gm})$ in most tissues as compared to oral or parenteral vaccinates. The virulent challenge strain was not recovered from any tissues collected from parenteral vaccinates at 4 weeks after experimental challenge. Although the challenge strain was recovered at low levels from some samples, tissue colonization in most tissues of oral vaccinates did not differ (P $>0.05)$ from parenteral vaccinates, but was reduced $(\mathrm{P}<0.05)$ when compared to colonization in non-vaccinated swine.
\end{abstract}

Keywords: Brucella swine; Brucellosis; Vaccine; Efficacy

\section{Introduction}

Brucellosis continues to be a significant zoonosis worldwide, with epidemiologic data suggesting that it remains a significant problem in many countries, and increasing or re-emerging in others [1]. Humans are effectively dead-end hosts and persistence of brucellosis requires maintenance in susceptible reservoir hosts. Several studies have demonstrated that addressing the pathogen in animal reservoirs is the most cost effective method for reducing human brucellosis [2,3].

Although new species of Brucella that are potentially zoonotic have been identified with reservoir hosts in wildlife [4-7], the three zoonotic species in domestic livestock are of much greater importance [8]. In order of virulence in humans, these species are: B. melitensis, B. suis, and B. abortus. Although preferred reservoir hosts for these Brucella species are sheep and goats, swine, and cattle, respectively [8], each has been demonstrated to be capable of infecting other host species. Numerous reports have identified B. suis as the cause of clinical disease in humans $[9,10]$. Additionally, B. suis is capable of infecting and inducing seropositivity in cattle $[11,12]$. In many countries, interest in B. suis is increasing due to its maintenance in expanding populations of feral swine or wild boar $[13,14]$. In the United States alone, an estimated 5 million feral swine are present and distributed over 39 states. Brucellosis in feral swine is widespread, especially in the southeastern United States, and seroprevalence rates may vary from state to state (range 0.3 to 52.6 percent). This large variation can be attributed to both true variations in disease prevalence and/or differences in serologic testing methods. Nevertheless, serological data suggest the widespread presence of B. suis in feral swine populations in the United States, and the potential for transmission to both humans and domestic animals.

Control of $B$. suis in feral swine populations is plagued with several challenges including lack of reliable serologic tests, and a rapidly expanding wildlife population across a wide geographic range. There are key differences in the pathogenesis of $B$. suis in swine, as compared to brucellosis (B. abortus or B. suis) in large or small ruminants [15]. Brucella suis in swine is characterized by a prolonged bacteremia and protracted shedding from mucosal surfaces as well as in urine. In addition, it has been previously demonstrated, that non-pregnant females and males are capable of shedding B. suis. Therefore, unlike other species 
of Brucella, males and non-pregnant female swine appear capable of contributing to $B$. suis transmission. In fact, zoonotic issues associated with processing of infected swine in abattoirs [16-18] are probably related to the persistent bacteremia and shedding from non-pregnant animals. Transmission mechanisms of brucellosis in feral swine are not completely characterized as both venereal and oral routes may contribute to infection. Oral exposure to aborted fetuses, infected placenta tissue, urine or even direct contact with contaminated surfaces cannot be excluded as significant routes for disease transmission. These materials may also pose a risk for the spread of B. suis to domestic animals and humans. Therefore, vaccination strategies that minimize shedding are of importance for reducing transmission of B. suis.

We have previously demonstrated that B. suis strain 3531 is a stable vaccine strain that is non-pathogenic in swine and induces humoral and cellular immunity after vaccination [19]. As 353-1 is a rough strain, it does not express the 0 side-chain on its lipopolysaccharide, and vaccinated swine remain seronegative on conventional brucellosis serologic tests after inoculation. In this study, we expand earlier work to determine efficacy of this vaccine in protecting feral swine against an experimental challenge with a virulent $B$. suis strain.

\section{Material and Methods}

\section{Vaccine Cultures}

Brucella suis strain 353-1 (353-1) was isolated from urine of a feral boar from Georgetown County, South Carolina (19). Characteristics of the isolate are included in Table 1 and the isolate was determined to be a Brucella suis biovar 1 by the National Veterinary Services Laboratory using standard diagnostic procedures. A master seed stock of 353-1 was prepared by propagation of a single colony from the original isolation plate on tryptose agar (Difco Laboratories, Detroit, MI) containing $5 \%$ bovine serum (TSA) at $37^{\circ} \mathrm{C}$ and $5 \% \mathrm{CO}_{2}$ for $72 \mathrm{hr}$. Bacteria were harvested using $0.15 \mathrm{M}$ phosphate buffered saline (PBS) and stored at $-80^{\circ} \mathrm{C}$ until vaccine or reagent preparation.

Brucella suis strain 3B, a virulent field strain first isolated from a boar in 1943, was obtained from the National Animal Disease Center culture collection.

For vaccination and experimental challenge of swine, strains 353-1 and 3B, respectively, were expanded on TSA for 48 hours at $37^{\circ} \mathrm{C}$ with $5 \% \mathrm{CO}_{2}$. Bacteria were harvested using PBS and diluted to a concentration of approximately $10^{10}$ colony-forming units (CFU) per ml for 353-1, or $10^{7} \mathrm{CFU} / \mathrm{ml}$ for strain 3B, using an optical density method and spectrophotometer (Molecular Devices, Sunnyvale, CA). Final concentrations of live bacteria in inoculums were determined by standard plate counts on TSA

For serology and lymphocyte proliferation assays, strain 3531 bacteria were grown on TSA for 48 hours at $37^{\circ} \mathrm{C}$, suspended in PBS, and bacterial concentrations determined by standard plate counts. After inactivation by incubation at $60^{\circ} \mathrm{C}$ for 2 hours, aliquots of the culture suspension were stored at $-80^{\circ} \mathrm{C}$.

\section{Vaccination}

Male and female, sexually intact feral swine, were captiveborn and raised at the United States Department of Agriculture/ Colorado State University Wildlife Research Facility in Fort Collins, Colorado, USA from a brucellosis-free herd. These animals were first and second generation offspring of feral swine that were originally obtained from the wild in Texas, USA and confirmed as brucellosis-free using serologic tests. After transport to our facility at 12 to 14 weeks of age, they were acclimated for 2 weeks in a bio level 3 containment facility, feral swine were randomly assigned, including blocking by sex, to parenteral $(n=8)$, oral $(n=8)$, and control $(n=9)$ treatments. For the duration of the experiment, feral swine were housed in the containment facility and segregated by treatment. Swine in vaccinated treatments received $2 \mathrm{ml}$ of phosphate buffered saline containing the live 353-1 strain, administered either intramuscular (parenteral) or into the caudal portion of the oral cavity (oral).

\section{Assessment of Tissue Colonization and In vivo Clearance}

At 8 weeks post-vaccination, 3 feral swine from both oral and parenteral vaccination groups were randomly selected and necropsied. Samples obtained at necropsy included lung, liver, spleen, lymphatic tissues (bronchial, hepatic, iliac, inguinal, mandibular, parotid, popliteal, prescapular, and retropharyngeal), blood, and urine.

\section{Assessment of Serologic Responses}

Blood samples were collected from parenteral- and orally-vaccinated feral swine by jugular venipuncture prior to vaccination, at $4,8,12$, and 17 weeks post-inoculation, and at 2 weeks after challenge. Non-vaccinated feral swine were

Table 1: Microbiologic Characteristics of Brucella suis strain 353-1. ${ }^{\text {a }}$

\begin{tabular}{|c|c|}
\hline Colonial Morphology & Rough \\
\hline Gram Stain & Gm- Coccobacilli \\
\hline Urease & Rapid positive \\
\hline Oxidase & Positive \\
\hline Catalase & Positive \\
\hline CO2 requirement & CO2 not required for growth \\
\hline H2S & Positive at 24 hours \\
\hline Antigens & A-, M-, R+ \\
\hline Lysis by Phages & R-, RTD- \\
\hline \multicolumn{2}{|c|}{ Growth in Media containing } \\
\hline Erythritol (2 mg/ml) & Positive \\
\hline Penicillin (5 $\mathrm{U} / \mathrm{ml})$ & Negative \\
\hline Thionine Blue (1:500) & Negative \\
\hline Thionin (1:25,000) & Positive \\
\hline Serum requirement & Negative \\
\hline Acriflavin agglutination & Positive \\
\hline
\end{tabular}

aTests performed in accordance with procedures described in Alton et al 1988. 
sampled at 4 and $8 \mathrm{wk}(\mathrm{n}=6)$ and at 2 weeks after experimental challenge $(n=9)$. Blood was allowed to clot for 12 hours at $4^{\circ} \mathrm{C}$ and centrifuged. Serum was divided into $1 \mathrm{ml}$ aliquots, frozen, and stored at $-70^{\circ} \mathrm{C}$.

Antibody responses against the vaccine strain of Brucella were determined by a previously described ELISA [20] in which heat-killed 353-1 is used as antigen with sera evaluated at a 1:100 dilution and results measured as optical density (OD) on a ELISA plate reader. Antibody responses prior to and at 4 weeks after experimental challenge were evaluated using a standard tube agglutination test [20].

\section{Post-Vaccination Lymphocyte Proliferation}

Blood was obtained from the jugular vein of parenteral and oral vaccinates at $4,8,12$, and 17 weeks after vaccination and placed into an acid-citrate dextrose solution. Non-vaccinated feral swine were only sampled at 4 and 8 weeks. Peripheral blood mononuclear cells (PBMC) were enriched by density centrifugation using a Ficoll-sodium diatrizoate gradient (Sigma Diagnostics, Inc., St. Louis, MO). Peripheral blood mononuclear cells were diluted in RPMI 1640 medium to $1 \times 10^{7}$ viable cells per $\mathrm{ml}$ as determined by trypan blue dye exclusion.

Fifty ul of each cell suspension, containing $5 \times 10^{5}$ cells, was added to each of two separate flat-bottom wells of 96-well microtiter plates that contained 100 ul of RPMI 1640 medium only, heat-killed $353-1$ ( $10^{5}$ to $10^{9}$ bacteria per well), or 1640 medium containing $1 \mathrm{ug} / \mathrm{ml}$ of pokeweed mitogen (PWM). Cell cultures were incubated for 7 days at $37^{\circ} \mathrm{C}$ in $5 \% \mathrm{CO}_{2}$. After a 7 day incubation, cell cultures were pulsed with $1.0 \mathrm{uCi}$ of $\left[{ }^{3} \mathrm{H}\right]-$ thymidine per well for 18 hours. Cells were harvested onto glass filter mats and counted for radioactivity in a liquid scintillation counter. Radioactivity was expressed as mean counts per minute (cpm), and stimulation indexes were determined by dividing the mean cpm from wells containing 353-1 antigen by the mean cpm from wells without antigen (RPMI 1640 medium only).

\section{Experimental B. suis Challenge}

At 18 weeks after inoculation, all remaining feral swine were intraconjunctivally challenged with $5 \times 10^{7} \mathrm{CFU}$ (50 ul of inoculum per eye) of B. suis strain 3B.

\section{Necropsy Procedures}

Swine were euthanized at 4 weeks after conjunctival challenge by intravenous injection of sodium pentobarbitol (Sleepaway, Ft. Dodge Labs, Ft. Dodge, IA, USA). Microbiologic samples obtained at necropsy after experimental challenge included: blood, urine, lymphatic tissues (bronchial, hepatic, inguinal, internal iliac, mandibular, messenteric, parotid, popliteal, prescapular, and retropharyngeal), uterus or epididymis/testis, spleen, liver, lung, kidney, and vaginal and conjunctival swabs.

\section{Bacterial Culture}

Tissue samples were processed in a similar manner as reported in other studies. Approximately $1 \mathrm{gm}$ of tissue samples were weighed and individually ground in $2 \mathrm{ml}$ of PBS ( $\mathrm{pH}=7.2$ ) using sterile glass grinders. Serial dilutions of each suspension of each tissue were plated onto TSA and Kuzda and Morse plates and incubated at $37^{\circ} \mathrm{C}$ in $5 \% \mathrm{CO}_{2}$ for 7 days. Brucella isolates were identified on the basis of colony morphology, growth characteristics, and a real time PCR assay using Brucella-specific primers and probe to the omp2A region of Brucella [20,21].

Blood was mixed 1:1 with tryptose broth (Difco Laboratories, Detroit, MI) containing 1\% sodium citrate. One $\mathrm{ml}$ from each blood culture was directly plated on tryptose agar containing $5 \%$ bovine serum. The remainder of each blood culture was held at $-5^{\circ} \mathrm{C}$ for 24 hours and then placed at $37^{\circ} \mathrm{C}$ with $5 \% \mathrm{CO}_{2}$ for $7,14,21$ and 28 days. At each time point, $1 \mathrm{ml}$ was taken from each culture and plated onto tryptose agar containing $5 \%$ bovine serum. As with tissue isolates, $B$. suis was identified after incubation at $37^{\circ}$ $\mathrm{C}$ and $5 \% \mathrm{CO}_{2}$ for 72 hours on the basis of colony morphology, growth characteristics, and a Brucella-specific PCR assay [20,21]

\section{Statistical Analysis}

Bacteriologic data are presented as mean \pm SEM. Serologic and colonization data were converted to the logarithm of the titer for analysis. Standard tube agglutination data which were negative on the first dilution and colonization data in which no recovery was made were converted to 1 for logarithmic conversion. Cell proliferation results were converted to stimulation indices (counts per minute (cpm) of wells containing antigen/cpm in absence of antigen) for statistical comparisons. Proliferation data from non-vaccinated feral swine prior to challenge were combined for analysis and compared to mean responses of vaccination treatments. Lymphoproliferative responses after vaccination, serologic data after vaccination and challenge, and tissue colonization after challenge were compared by general linear model procedure (SAS Institute Inc., Cary, NC, USA) and presented as mean \pm SEM. Means were separated by a least square means procedure $(P<0.05)$. Fisher's exact test was used to evaluate differences in incidence of infection between treatment groups.

\section{Animal Welfare}

The animal experiments as performed in this study were approved by the National Animal Disease Center Institutional Animal Care and Use Committee. Procedures and biocontainment were in accordance with requirements of the Select Agent Act.

\section{Results and Discussion}

The overall results of our study suggest that parental and oral vaccination of feral swine with strain 353-1 was effective in protecting against infection after experimental challenge with a virulent strain of $B$. suis.

Antibody titers against $B$. suis strain 353-1 and from parenteral and oral vaccinates were greater $(P<0.05)$ at all sampling times after vaccination when compared to titers from non-vaccinated feral swine (Figure 1). Titers between parenteral and oral vaccinate groups did not differ $(P>0.05)$ when compared to each other. Two weeks after experimental challenge of feral swine, titers from parenteral and oral vaccinates to strain 353-1 
did not differ $(P>0.05)$, but continued to be significantly higher $(P<0.05)$ than responses of control pigs. Altogether, these data suggest that both oral and parenteral vaccination with Brucella strain 353-1 promote strong and sustained antibody responses.

Field detection of brucellosis is primarily based on serologic testing. For detection of brucellosis in swine, current serum agglutination tests lack both sensitivity and specificity, which affects their ability to accurately determine the true prevalence of brucellosis within herds or populations. An ideal vaccine would be efficacious while also not interfering with serologic testing; thereby allowing discrimination between infected and vaccinated animals. We used the standard tube agglutination test to monitor the serological status of our experimental animals Prior to experimental challenge, sera from all groups were negative on the standard tube agglutination test and mean titers did not differ $(P>0.05)$ between treatments. After experimental challenge, control pigs had greater $(P<0.05)$ mean standard tube agglutination titers $(53.1 \pm 8.0)$ as compared to mean titers of swine in the parenteral $(0 \pm 0)$ or oral $(12.5 \pm 12.5)$ vaccination treatments. As expected, these data indicate that vaccination with strain 353-1 did not cause positive serologic responses that would interfere with detecting Brucella-infected swine.

Cellular immune responses are believed to be the primary immunologic mechanism for protection against intracellular pathogens. In the current study, proliferative cellular immune responses were elicited after parenteral and oral vaccination. When compared to non-vaccinated feral swine, parenteral vaccinates had greater $(P<0.05)$ proliferative responses to Brucella antigens at 12 and 17 weeks after vaccination. In the current study, proliferative responses of oral vaccinates did not differ $(P>0.05)$ from responses of parenteral vaccinates at 12 and 17 weeks, but differed $(P<0.05)$ from responses of control animals only at 12 weeks (Figure 2). The induction of cellular immunity in swine is consistent with data from our laboratory in which vaccinated cattle and bison demonstrate significant cellular responses beginning at 12 to 16 weeks after parenteral vaccination, Based on these data, both parenteral and

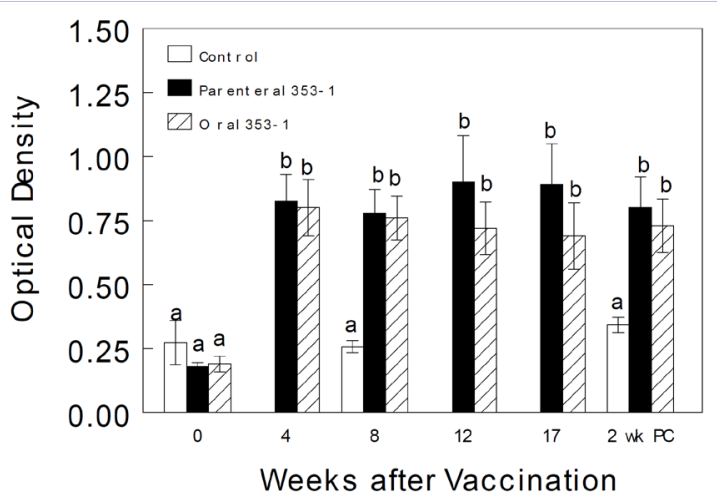

Figure 1: Serologic responses of feral swine to $\gamma$-irradiated 353-1 in am ELISA assay after vaccination with saline, or after parenteral or oral vaccination with $1.9 \times 10^{10} \mathrm{CFU}$ of strain 353-1. Responses are presented as mean optical density \pm SEM. Means with different superscripts are significantly different $(P<0.05)$.

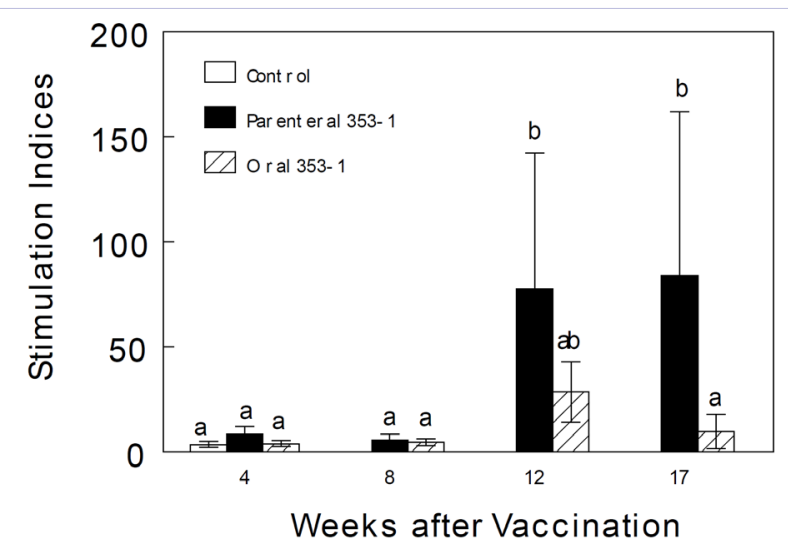

Figure 2: Proliferative responses to $10^{7}$ to $10^{9} \mathrm{CFU}$ of $\gamma$-irradiated 3531 by peripheral blood mononuclear cells from feral swine vaccinated with saline, or after parenteral or oral vaccination with $1.9 \times 10^{10} \mathrm{CFU}$ of strain 353-1. Cells were incubated at $37^{\circ} \mathrm{C}$ and $5 \% \mathrm{CO}_{2}$ for 7 days and pulsed for $18 \mathrm{hrs}$ with $\left[{ }^{3} \mathrm{H}\right]$-thymidine. Results are expressed as mean stimulation indices \pm SEM. Means within a sampling time with different superscripts are significantly different $(P<0.05)$.

oral vaccination with strain 353-1 induced cellular proliferative responses in swine; with parenteral vaccination appearing to have induced more robust responses.

Presence of the vaccine strain was evaluated at 8 weeks postvaccination by culturing urine and tissues collected at necropsy from oral and parenteral vaccinated pigs. In the oral vaccination group, the vaccine strain was only recovered in urine from 1 pig and from lymph nodes of 2 of 3 animals (Data not shown). In contrast, the vaccine strain was not recovered from any sample obtained from three parenteral vaccinated swine (Data not shown). Although numbers of animals were limited, data from this study and others [19] suggests rapid in vivo clearance of strain 353-1 after vaccination of swine.

After experimental challenge, blood and swabs (conjunctival and vaginal) collected at necropsy were negative for isolation of B. suis in all animals.

Because the challenge strain was not recovered from any sample obtained at necropsy from parenteral vaccinates, incidence of recovery of $B$. suis from parenteral vaccinates was reduced $(P<0.05)$ when compared to recovery from nonvaccinated swine (Table 2 ). In a similar comparison, the incidence of recovery of virulent $B$. suis from oral vaccinates was reduced $(P<0.05)$ in all tissue groups except cervical lymph nodes, when compared to recovery from swine in the control treatment.

When colonization of tissues were compared across treatments (Table 3), parenteral or oral vaccination with 3531 reduced CFU/gm of virulent $B$. suis in lung, spleen, and most lymphatic tissues (bronchial, mandibular, mesenteric, parotid, prescapular, popliteal, iliac, and inguinal lymph nodes). However, mean colonization in liver, kidney, hepatic, and retropharyngeal lymph nodes did not statistically differ $(P>0.05)$ between nonvaccinated and vaccinated treatments. A statistical difference $(P$ $<0.05$ ) between treatments in mean colonization was found in 
Table 2: Recovery of $B$. suis from tissues obtained at necropsy after conjunctival challenge with $B$. suis strain $3 \mathrm{~B}$ of feral swine vaccinated with $10^{10}$ colony-forming units of B. suis strain 353-1.

\begin{tabular}{|c|c|c|c|}
\hline & & Parenteral & Oral \\
\hline & Control & Vaccinates & Vaccinates \\
\hline \multicolumn{4}{|c|}{ Feral Swine } \\
\hline Major Organs ${ }^{\mathrm{a}}$ & $9 / 9$ & $0 / 5^{*}$ & $0 / 5^{*}$ \\
\hline $\begin{array}{l}\text { Reproductive } \\
\text { Tissues }^{\mathrm{b}}\end{array}$ & $8 / 9$ & $0 / 5^{*}$ & $0 / 5^{*}$ \\
\hline $\begin{array}{l}\text { Cervical Lymph } \\
\text { Nodes }^{c}\end{array}$ & $7 / 9$ & $0 / 5^{*}$ & $2 / 5$ \\
\hline $\begin{array}{l}\text { Other Lymph } \\
\text { Nodes }^{\mathrm{d}}\end{array}$ & $9 / 9$ & $0 / 5^{*}$ & $2 / 5^{*}$ \\
\hline
\end{tabular}

${ }^{a}$ Major organs are defined as lung, liver, spleen, and kidney.

${ }^{b}$ Reproductive tissues are defined as uterus, testes, epididymis, and illiac or inguinal lymph nodes.

${ }^{c}$ Cervical lymph nodes are defined as parotid, mandibular, or retropharyngeal.

dOther lymph nodes are defined as bronchial, hepatic, mesenteric, popliteal, and prescapular.

- Denotes incidence of infection in the group of tissues for animals in a vaccination treatment is less $(P<0.05)$ than incidence in the nonvaccinated treatment.

Table 3: Colonization (colony-forming units/gm) of B. suis in target tissues at 4 weeks after experimental $B$. suis challenge of feral swine vaccinated with $10^{10}$ colony-forming units of $B$. suis strain 353-1.

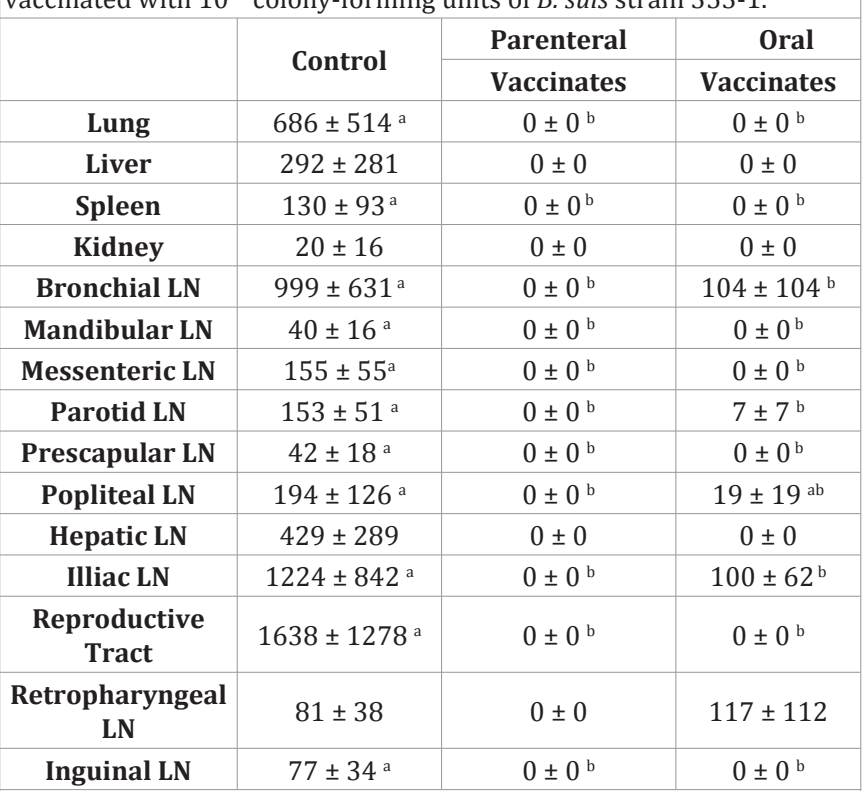

Data are presented as mean colony-forming units (CFU) per gm of tissue.

Treatment means within a tissue with different superscripts are statistically $(P<0.05)$ different.

most, but not all tissues.

Gender did not influence recovery $(P>0.05)$ of the challenge strain at necropsy. For example, in reproductive tissues (uterus, testes, epididymis, inguinal lymph node, or iliac lymph node) of non-vaccinates, the challenge strain was recovered from tissues of five females and three males. It should be emphasized that the 353-1 vaccine strain was not recovered at necropsy after experimental challenge.

Overall, the observed high colonization of the challenge strain in tissues from control feral swine, and the absence, or significant reduction of colonization in vaccinates, suggests the 353-1 vaccine is efficacious against $B$. suis in swine. While oral vaccination with strain 353-1 was not as efficacious as parenteral vaccination, it should be emphasized that reproductive tissues for both vaccination groups were culture negative, implying that transmission of brucellosis would be reduced by vaccination. Therefore, vaccination with the 353-1 strain could reduce bacterial colonization and shedding in swine, leading to reduced transmission within feral swine populations and to other potential hosts (humans or domestic livestock).

Capture of a significant portion of feral swine populations for vaccination is an unrealistic expectation. Populations of feral swine are large and dispersed across wide areas, and the disposition and intelligence of feral swine make entrapment difficult. Therefore, a brucellosis vaccine that could be orally delivered under natural conditions would be ideal for addressing brucellosis in feral swine or wild boar. Although data from our study suggests that oral vaccination of feral swine with 353-1 is not as efficacious as parenteral vaccination, our results indicate that oral delivery could be beneficial for brucellosis control. It would be of interest to determine if tissue colonization in oral vaccinates would have demonstrated greater reductions if the time between challenge and necropsy was increased, or if booster vaccination was utilized.

It should be noted that experimental challenge models used for evaluation of efficacy of brucellosis vaccines in swine need further characterization to define the relationship between infection and reproductive effects, so that clinical disease after experimental challenge replicates the epidemiologic features of B. suis under field conditions. The experimental challenge used in this study utilized a virulent strain from published studies dating back in the 1950's [22]. Virulence of this strain was also supported by data obtained in previous studies in which in vivo persistence of this strain after conjunctival inoculation was characterized in naïve pigs (Olsen, unpublished). The observed high rate of infection in non-vaccinated swine after experimental infection in the current study was similar to the prevalence of Brucella infection in feral swine under field conditions [23]. In that study, the high recovery rate $(77.5 \%)$ of Brucella in randomly trapped feral swine most likely reflects the epidemiologic features of brucellosis in a chronically infected feral swine population. Our challenge model is also supported by data that indicates that prepubescent swine, as used in the current study, can be readily infected and may demonstrate persistent infections for up to 144 days $[14,16,22,24,25]$.

We have previously demonstrated that $B$. suis strain 353-1 is a natural rough mutant, is genetically stable, does not induce positive responses on brucellosis surveillance tests, is not shed 
after vaccination, and is clinically safe in swine [19]. Based on these characteristics and the efficacy data presented in this paper, strain 353-1 appears to be a promising vaccine for addressing brucellosis in swine that will not impair serologic surveillance of B. suis. Although additional studies are needed, our data suggests that oral delivery of this vaccine could be an effective strategy for increasing protection in swine against $B$. suis, thereby making it ideal for delivery to wild boar and feral swine.

\section{Acknowledgements and Declarations}

The authors thank Aileen Bryant, Katrina Pille, Doug Ewing, Dennis Johannes, Dave Lubbers, Rebecca Madison, Darl Pringle, Jay Steffen, and Robin Zeisneiss for their technical assistance. Funding for this study was provided by budget appropriations within the U.S. government to the Agricultural Research Service.

Names are necessary to report factually on available data; however, the USDA neither guarantees nor warrants the standard of the product, and the use of the name by USDA implies no approval of the product to the exclusion of others that may also be suitable.

\section{References}

1. Rubach MP, Halliday JE, Cleaveland S, Crump JA. Brucellosis in low-income and middle-income countries. Curr Opin Infect Dis. 2013;26(5):404-12

2. Bernués A, Manrique E, Maza MT. Economic evaluation of bovine brucellosis and tuberculosis eradication programmes in a mountain area of Spain. Prev Vet Med. 1997;30(2):137-149.

3. Roth F, Zinsstag J, Orkhon D, Chimed-Ochir G, Hutton G, Cosivi O, et al Human health benefits from livestock vaccination for brucellosis: case study. Bull World Health Organ. 2003;81(12):867-876.

4. Scholz HC, Hofer E, Vergnaud G, Le Fleche P, Whatmore AM, Al Dahouk $\mathrm{S}$, et al. Isolation of Brucella microti from mandibular lymph nodes of red foxes, Vulpes vulpes, in lower Austria. Vector Borne Zoonotic Dis. 2009;9(2):153-156.

5. Scholz HC, Nõckler K, Gõlner C, Bahn P, Vergnaud G, Tomaso H, et al. Brucella inopinata sp. nov. isolated from a breast implant infection. Int J Syst Evol Microbiol. 2010;60(Pt 4):801-808.

6. Cloeckaert A, Bernardet N, Koylass MS, Whatmore AM, Zygmunt MS Novel IS711 chromosomal location useful for identification of marine mammal Brucella genotype ST27, which is associated with zoonotic infection. J Clin Microbiol. 2011;49(11):3954-3959.

7. Hofer E, Revilla-Fernandez S, Al Dahouk S, Riehm JM, Nõckler K, Zygmunt MS, et al. A potential novel Brucella species isolated from mandibular lymph nodes of red foxes in Austria. Vet Microbiol. 2012;155(1):93-99.

8. Godfroid J, Scholz HC, Barbier T, Nicolas C, Wattiau P, Fretin D, et al. Brucellosis at the animal/ecosystem/human interface at the beginning of the 21st century. Prev Vet Med. 2011;102(2):118-131.

9. Meirelles-Bartoli RB, Mathias LA, Samartino LE. Brucellosis due to Brucella suis in a swine herd associated with a human clinical case in the state of Sao Paulo, Brazil. Trop Anim Health Prod. 2012;44(7):15751579.

10. Naha K, Dasari DS, Pandit V, Seshadri S. A rare case of seronegative culture-proven infection with Brucella suis. Australas Med J. 2012;5(7):340-343

11. Ewalt DR, Payeur JB, Rhyan JC, Geer PL. Brucella suis biovar 1 in naturally infected cattle: a bacteriological, serological and histological study. J Vet Diagn Invest.1997;9(4):417-410

12. Szulowski K, Iwaniak W, Weiner M, Zlotnicka J. Brucella suis biovar 2 isolations from cattle in Poland. Ann Agric Environ Med. 2013;20(4):672-675.

13. De Massis F, Di Provvido A, Di Sabatino D, Di Francesco D, Zilli K, Ancora M, et al. Isolation of Brucella suis biovar 2 from a wild boar in the Abruzzo Region of Italy. Vet Ital. 2012;48(4):397-404.

14. Musser JMB, Schwartz AL, Srinath I, Waldrup KA. Use of serology and bacterial culture to determine prevalence of Brucella spp. in feral swine (Sus scrofa) in proximity to a beef cattle herd positive for Brucella suis and Brucella abortus. J Wildl Dis. 2013;49(2):215-220.

15. Hutchings LM. Report of further studies of brucellosis in swine. Proceedings of the 48th Annual Meeting of the U.S. Livestock Sanitary Association.1944;105-109.

16. Deyoe BL, Manthei CA. Sties of localization of Brucella suis in swine. Proceedings of the 71st Annual Meeting of the. U.S. Livestock Sanitary Association.1967;102-108.

17. Huddleson F, Johnson HW, Hamann EE. A study of Brucella infection in swine and employees of packing-houses. J Am Vet Med Assoc. 1933;83:16-30.

18. Trout D, Gomez TM, Bernard BP, Mueller CA, Smith CG, Hunter L, et al. Outbreak of brucellosis at a United States pork packing plant. J Occup Environ Med. 1995;37(6):697-703.

19. Stoffregen WC, Johnson CS, Olsen SC. Immunogenicity and safety of a natural rough mutant of Brucella suis as a vaccine for swine. Res Vet Sci. 2-013;95(2):451-458

20. Lee IK, Olsen SC, Bolin CA. Effects of exogenous recombinant interleukin-12 on immune responses and protection against Brucella abortus in a murine model. Can J Vet Res. 2001; 65(4):223-228.

21. Alton GG, Jones LM, Angus RD, Verger JM. Bacteriologic methods and serologic methods. In: Techniques for the brucellosis laboratory. Institut National de la Recherche Agronomique. Paris, France. $1988 ; 190$

22. Manthei CA, Mingle CK, Carter RW. Brucella suis infection in suckling and weanling pigs I. J Am Vet Med Assoc. 1952;121(908):373-82.

23. Stoffregen WC, Olsen SC, Wheeler JC, Bricker BJ, Palmer MV, Jensen $\mathrm{AE}$, et al. Diagnostic characterization of a feral swine herd enzootically infected with Brucella. J Vet Diagn Invest. 2007;19(3):227-237.

24. Manthei CA. Research on swine brucellosis by the Bureau of Animal Industry (1941-1947). Am J Vet Res. 1948;9:40-45.

25. Hutchings LM, Delez AL, Donham CR. Studies on brucellosis of swine: I. Infection experiments with weanling pigs. Am J Vet Res.1944;5:195208. 